Módulo Cero y su impacto positivo en el aprendizaje del idioma Inglés como lengua extranjera en los alumnos del Primer Semestre de la Universidad Estatal de Milagro

Irlanda Maridueña Macancela, Iliana Real Poveda 


\title{
Módulo Cero y su impacto positivo en el aprendizaje del idioma inglés como lengua extranjera en los alumnos del Primer Semestre de la Universidad Estatal de Milagro
}

\author{
Irlanda Jacqueline Maridueña Macancela, Iliana Verónica Real Poveda, MAE \\ Docentes - Universidad Estatal de Milagro \\ jacquemar4@gmail.com; ilianarealpoveda@gmail.com
}

\begin{abstract}
Resumen
El idioma Inglés se ha convertido en una de las más grandes oportunidades y debilidades de las personas en el mundo entero, puesto que no todos pueden desarrollar las diferentes destrezas de esta lengua extranjera, lo cual ha causado desacierto al momento de comprender este idioma, rechazando la asignatura como tal, evitando la adquisición del mismo como herramienta básica en este mundo globalizado. Este trabajo investigativo surgió de la necesidad de evidenciar la importancia de contar con el Módulo Cero dirigido a los estudiantes que ingresan a la Universidad Estatal de Milagro, trabajo que se realizó considerando los métodos cuantitativo y cualitativo tomando como muestra a 65 estudiantes que aprobaron el Módulo Cero en el Primer Semestre 2015. Las autoras reiteran la importancia de este Módulo Cero de Inglés, ya que los resultados demostraron que los alumnos adquirieron los conocimientos necesarios acordes con su nivel para avanzar al Módulo 1 de Inglés. El aprendizaje progresivo de este idioma extranjero servirá para estrechar lazos de confianza y adaptación entre estudiantes y docentes haciendo más placentera la adquisición de esta lengua para que los educandos alcancen el dominio y la facilidad de comunicarse en Inglés en los distintos campos de estudio.
\end{abstract}

Palabras clave: módulo Cero, aprendizaje del idioma Inglés

\begin{abstract}
The English language has become one of the greatest opportunities and weaknesses of people worldwide, since not everyone can develop the different skills of this foreign language, which has caused uncertainty at the moment of understanding it, rejecting the subject and therefore, avoiding the acquisition of it as a basic tool in this globalized world. This research work arose from the need to demonstrate the importance of having the Zero Module addressed to students who enter to the State University of Milagro; this work was done considering the quantitative and qualitative methods which were developed taking as a sample 65 students who approved The Zero Module in the First Semester 2015. The authors reiterate the importance of this English Starter Module, since the results demonstrated that the students acquired the necessary knowledge according to their level to continue to Module 1 of English. The progressive learning of English as a foreign language will serve to strengthen ties of trust and adaptation between students and teachers making it more enjoyable to acquire this language, besides learners will achieve proficiency and ease of communicating in English in the different academic spheres.
\end{abstract}

Keywords: module zero - English language learning 


\section{Introducción}

En la actualidad la relevancia del idioma inglés se ha estandarizado en todos los ámbitos y establecimientos educativos del Ecuador y de todos los países de habla hispana, debido a ello, cada vez es mayor el número de universidades y Escuelas Politécnicas que requieren este idioma ya sea para el ingreso o salida de los estudiantes. Esto hace que se considere al idioma inglés como una herramienta de comunicación global que dirige al acceso de amplios conocimientos con tecnología que benefician al entorno y la sociedad. (Superior, Consejo de Educación Superior - República del Ecuador, 2013) [1]

El idioma inglés permite generar la comprensión de la propia lengua y cultura, ampliando el desarrollo de las habilidades cognitivas de la comunidad. (Uribe Zirene, 2012). [2]

De esta manera, el aprendizaje del idioma inglés constituye un limitante para muchos estudiantes que ingresan a la Universidad Estatal de Milagro y demuestran inconformidad y falta de importancia en la asignatura, lo cual se ve reflejado en el proceso, desarrollo y resultados de los módulos de inglés que se dictan en este Centro de Educación Superior. (Maridueña Macancela, 2014) [3]

A partir del Primer Semestre 2015, el Sistema Modular de Inglés dio la apertura al Módulo Cero para homogenizar conocimientos de los estudiantes que ingresan a las diferentes carreras de estudio en la Universidad Estatal de Milagro (UNEMI), proceso que fue aprobado por el Órgano Colegiado Académico Superior, mediante Resolución OCAS-SO-14-2014. (Milagro, Resoluciones OCAS 2014 - UNEMI, 2014) [4]

Para llevar a efecto este proceso se receptó una prueba de suficiencia del idioma inglés a los estudiantes que ingresaron por primera vez a la UNEMI, es decir, a quienes aprobaron el Pre-Universitario, a fin de medir sus conocimientos y ubicarlos en el nivel correspondiente, sea esto Módulo Cero o Módulo Uno (Fleta Guillén, 2006). Se consideró una escala de valoración del 01 al 44 para el Módulo Cero y del 45 al 100 para el Módulo Uno, respectivamente.

En la prueba de suficiencia se consideró:
a) Use of English
(50 puntos)
b) Listening
(10 puntos)
c) Reading
(20 puntos)
d) Writing
(20 puntos)

La tabla que se detalla a continuación muestra de manera general el número de estudiantes que ingresaron a estos dos grupos de módulos:
Tabla 1

\begin{tabular}{|c|c|c|c|}
\hline Facultad & Carrera & $\begin{array}{c}\text { Nivel } \\
\text { Cero } \\
\text { (Nivel de } \\
\text { inicio) } \\
\end{array}$ & Nivel 1 \\
\hline \multirow{4}{*}{$\begin{array}{l}\text { Ciencias Administrativas } \\
\text { Comerciales }\end{array}$} & Ingeniería Comercial & 26 & 19 \\
\hline & Ingeniería C.P.A. & 33 & 24 \\
\hline & Ingeniería Marketing & 28 & 8 \\
\hline & Turismo & 14 & 5 \\
\hline \multirow{3}{*}{$\begin{array}{l}\text { Ciencias de la Educación y la } \\
\text { Comunicación }\end{array}$} & Psicología & 13 & 23 \\
\hline & C. Social & 22 & 1 \\
\hline & Diseño Gráfico & 9 & 10 \\
\hline \multirow[t]{2}{*}{ Ciencias de la Ingeniería } & $\begin{array}{l}\text { Ingeniería en } \\
\text { Sistemas }\end{array}$ & 30 & 16 \\
\hline & Ingeniería Industrial & 32 & 18 \\
\hline \multirow{3}{*}{ Ciencias de la Salud } & Enfermería & 33 & 34 \\
\hline & Terapia Respiratoria & 16 & 7 \\
\hline & Nutrición Humana & 19 & 12 \\
\hline \multicolumn{2}{|c|}{ Exonerados (diferentes carreras) } & & 59 \\
\hline & Total: & 275 & 236 \\
\hline
\end{tabular}

Fuente: Resultados de la prueba de suficiencia Pre-Universitario (SMI)

Es importante mencionar que existió un incremento de 50 alumnos al Módulo Cero, quienes homologaron materias de otras universidades pero necesitaban empezar sus estudios de Inglés, con esto se forma el total de 325 estudiantes que realizaron el Módulo Cero en el Primer Semestre 2015.

Se abrieron 12 cursos o módulos ceros en diferentes horarios y fueron asignados 4 docentes para trabajar con estos grupos debiendo realizar una nueva programación acorde con el texto American English File Starter, el mismo que se utilizó para dictar las clases a los estudiantes del Módulo Cero. (LathamKoening C, Oxenden C, y Seligson P, 2013) [5]

\section{Importancia del problema}

Luego de haber concluido el Primer Semestre 2015, de acuerdo a los resultados obtenidos gracias al Sistema de Gestión Académica (SGA) de la Universidad Estatal de Milagro, se detalla en la siguiente tabla la información al respecto, pudiéndose apreciar que de los 325 alumnos en total que ingresaron a este Módulo Cero, 198 aprobaron el módulo con conocimientos del idioma Inglés, lo cual les permitirá aprovechar al máximo lo aprendido para evidenciarlo en el Módulo 1. De igual manera, se constató que 127 alumnos reprobaron el módulo cero probablemente debido al poco interés en el curso o no asistieron al mismo. (Milagro, sga.unemi, 2015) [6]

Sin embargo, existe una Resolución del Órgano Colegiado Académico Superior OCAS-SO-14-2014, que indica que los estudiantes que reprobaron el Módulo Cero pueden inscribirse al Módulo 1 en este Segundo Semestre 2015. Es decir, que quedará en los usuarios demostrar interés para aprobar el módulo cero. (Milagro, Resoluciones OCAS 2014 - UNEMI, 2014)

A pesar de que los resultados indican que existieron alumnos que no aprobaron el Módulo Cero, esta información genera mucha expectativa con los 
estudiantes que lograron aprobar el nivel y que se encuentran realizando el módulo 1, puesto que se entiende que harán un buen desempeño durante el mismo. A continuación se detalla la información referente a los 12 módulos Inglés Cero que se dictaron en el Primer Semestre 2015:

Tabla 2

\begin{tabular}{|c|c|c|c|}
\hline Docente & Total & Aprobado & Reprobado \\
\hline \multirow{3}{*}{ Choez Menoscal César Eduardo, MSc. } & 33 & 11 & 22 \\
\cline { 2 - 4 } & 33 & 17 & 16 \\
\cline { 2 - 4 } & 33 & 9 & 24 \\
\hline \multirow{3}{*}{ Garda Brito Oswaldo Edison, MSc } & 13 & 10 & 3 \\
\cline { 2 - 4 } & 34 & 30 & 4 \\
\cline { 2 - 4 } & 26 & 17 & 9 \\
\hline \multirow{3}{*}{ Ledesma Acosta Brigita Valerina, MAE } & 31 & 20 & 11 \\
\hline & 24 & 17 & 7 \\
\cline { 2 - 5 } & 23 & 9 & 14 \\
\cline { 2 - 5 } & 33 & 26 & 7 \\
\cline { 2 - 5 } & 24 & 22 & 2 \\
\cline { 2 - 5 } & 18 & 10 & 8 \\
\hline \multirow{3}{*}{ Moreno Cartagena Gladys Vanessa, MSc. } & 325 & 198 & 127 \\
\hline & & & \\
\hline
\end{tabular}

Fuente: Sistema Modular de Inglés

En el Segundo Semestre 2015, la Coordinación del Sistema Modular de Inglés volvió a receptar este tipo de prueba de Suficiencia del Idioma Inglés. Se detalla la siguiente información relacionada al número de estudiantes que fueron ubicados al Modulo Cero o de Inicio y al Módulo I de Inglés:

Tabla 3

\begin{tabular}{|c|c|c|c|}
\hline \multirow{2}{*}{ Facultad } & Carrera & $\begin{array}{c}\text { Módulo Cero } \\
\text { o de Inicio }\end{array}$ & Inglés I \\
\hline \multirow{4}{*}{ Ciencias Administrativas y Comerciales } & $\begin{array}{c}\text { Ingeniería } \\
\text { Comercial }\end{array}$ & 24 & 24 \\
\cline { 2 - 4 } & Ingeniería C.P.A. & 27 & 22 \\
\cline { 2 - 4 } & Turismo & 18 & 24 \\
\hline Ciencias de la Educación y la & Psicología & 12 & 16 \\
\cline { 2 - 5 } Comunicación & C. Social & 12 & 8 \\
\hline \multirow{3}{*}{ Ciencias de la Ingeniería } & $\begin{array}{c}\text { Ingeniería en } \\
\text { Sistemas }\end{array}$ & 31 & 32 \\
\cline { 2 - 5 } & $\begin{array}{c}\text { Ingeniería } \\
\text { Industrial }\end{array}$ & 34 & 50 \\
\hline \multirow{2}{*}{ Ciencias de la Salud } & Enfermería & 47 & 73 \\
\cline { 2 - 5 } & $\begin{array}{c}\text { Nutrición } \\
\text { Humana }\end{array}$ & 17 & 24 \\
\hline \multicolumn{2}{|c|}{ Total: } & 222 & 280 \\
\hline
\end{tabular}

Fuente: Resultados de la prueba de suficiencia Pre-Universitario

(SMI)

De igual manera, en el Primer Semestre 2016, luego de receptar la prueba de suficiencia a 554 estudiantes del semestre en mención, se obtuvo el siguiente resultado:

\begin{tabular}{|c|c|c|}
\hline Facultad & $\begin{array}{c}\text { Nivel Cero } \\
\text { (Nivel de } \\
\text { inicio) }\end{array}$ & Nivel 1 \\
\hline Ciendias Administrativas y Comerciales & 88 & 63 \\
\hline Ciencias de la Educación y la Comunicación & 95 & 47 \\
\hline Ciencias de la Ingeniería & 92 & 59 \\
\hline Ciencias de la Salud & 59 & 51 \\
\hline Total: & 334 & 220 \\
\hline
\end{tabular}

Fuente: Resultados de la prueba de suficiencia Pre-Universitario (SMI)
En este Segundo Semestre 2016, el Sistema Modular de Inglés procedió a receptar la prueba de suficiencia a 744 alumnos para verificar el nivel de Inglés de los estudiantes que ingresan a la Universidad Estatal de Milagro y que vienen de los diferentes establecimientos educativos del país y la región. Seguidamente se detallan los resultados:

Tabla 5

\begin{tabular}{|c|c|c|}
\hline Facultad & $\begin{array}{c}\text { Nivel Cero } \\
\text { (Nivel de } \\
\text { inicio) } \\
\end{array}$ & Nivel 1 \\
\hline Ciencias Administrativas y Comerdales & 103 & 89 \\
\hline Ciencias de la Educación y la Comunicación & 170 & 118 \\
\hline Ciencias de la Ingeniería & 99 & 70 \\
\hline Ciencias de la Salud & 44 & 51 \\
\hline Total: & 416 & 328 \\
\hline
\end{tabular}

Luego de haber llevado la secuencia de estos cuatro semestres de estudio, se analiza el nivel de dispersión que existe entre los 2 niveles como resultado de la prueba de suficiencia, lo cual referencia la escala exponencial de la misma.

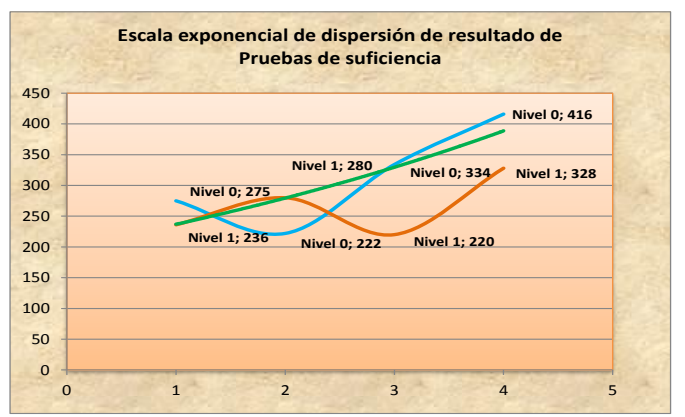

Figura 1. Escala exponencial de dispersión de resultado de Pruebas de suficiencia

Para emitir criterio de manera separada en torno a los 2 niveles de Inglés como resultado de la prueba de suficiencia receptada a los estudiantes que ingresan al Primer Semestre, se presenta lo siguiente:

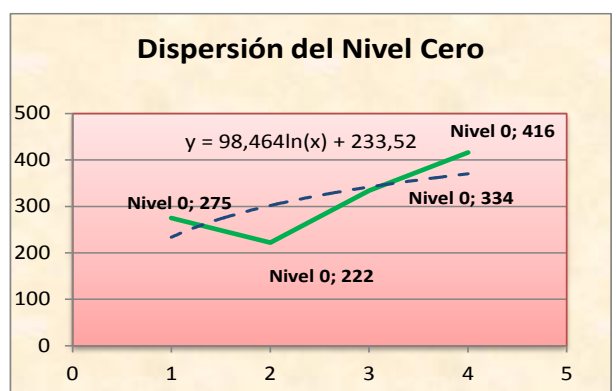

Figura 2. Dispersión del Nivel Cero

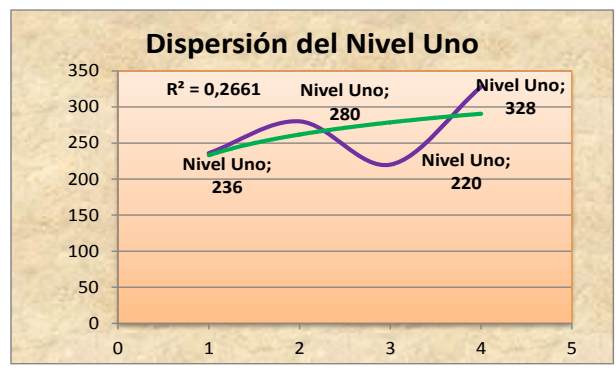

Figura 3. Dispersión del Módulo Uno 
De acuerdo a la información que se registra en el gráfico de dispersión del Nivel Cero, en el primer semestre 2015, el $22 \%$ de los alumnos realizaron el módulo cero. En el segundo semestre 2015, el $18 \%$ de los estudiantes cursaron este módulo de inicio. De igual manera, en el primer semestre 2016, el 27\% de quienes ingresaron a este centro de educación superior fueron quienes realizaron el mencionado módulo Cero. Finalmente, en este segundo semestre 2016, se presentó el incremento del 33\% que se encuentran cursando el módulo cero. (Fleta Guillén, 2006) [7]

Por lo tanto, es elocuente observar que la tendencia del nivel cero se ha incrementado y ello hace referenciar la importancia de seguir dictando este módulo cero de Inglés a los estudiantes que ingresan al primer semestre de la Universidad Estatal de Milagro, ya que durante este tiempo pueden reforzar el conocimiento de la lengua inglesa y de esta manera, evitar tener confusiones en la continuidad de los módulos reglamentarios de Inglés. (Navas Yonaiker; Real Iliana; Pacheco Silvia, 2016) [8]

\subsection{Programa del Módulo Cero}

El Módulo Cero consiste en la programación que sirve específicamente para reforzar los conocimientos y destrezas del idioma inglés a favor de los alumnos que ingresan al Primer Semestre en esta universidad, de tal manera que al momento de ingresar al Nivel 1, ya no tengan vacíos en este idioma.

Esta disposición dirigida por el OCAS se aplica en virtud de los vacíos que presentan los estudiantes que ingresan a la Universidad Estatal de Milagro en cuanto a la asignatura de Inglés y esa es una de las causas por las que los alumnos reprueban o se retiran de los módulos de Inglés puesto que el texto que se usa en el Módulo 1, es Nivel Elemental, lo cual ellos desconocen. Por ello la importancia del Módulo Cero ya que se utiliza un texto de Nivel de Principiante conforme a las necesidades de los estudiantes. (Milagro, Resoluciones OCAS 2014 - UNEMI, 2014)

Se debe tener presente que el nivel de Inicio no es otra cosa que el Nivel A1 de acuerdo al Marco Común Europeo de referencia para las lenguas: (Assessment, 2015)

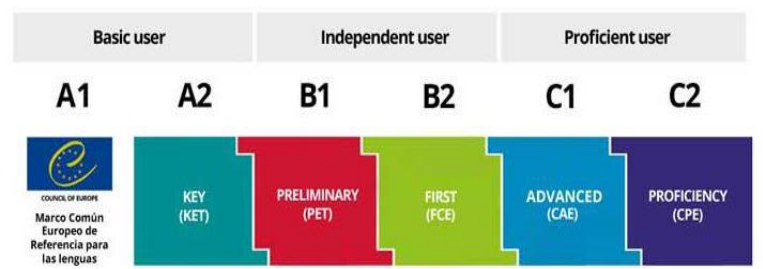

Figura 4. Niveles acorde con el Marco Común Europeo. Fuente: Cambridge University Press

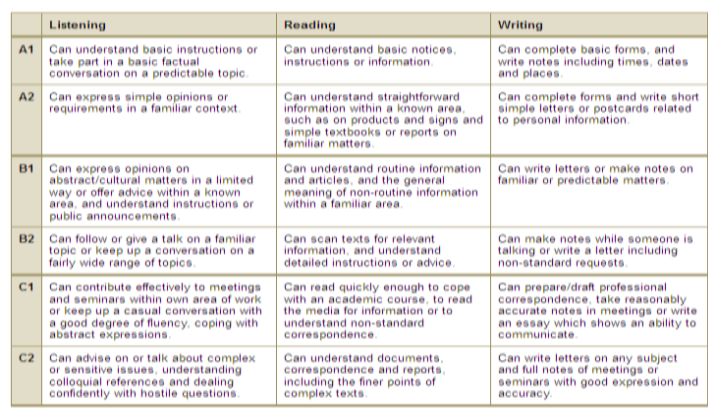

Figura 5. Desarrollo de destrezas Fuente: Cambridge University Press

De acuerdo a esta tabla referente a la escala de niveles del idioma Inglés del Marco Común Europeo de referencia para las lenguas, se explica notablemente que al término del Módulo Cero, los alumnos tendrán la habilidad para:

$\checkmark \quad$ Es capaz de comprender instrucciones básicas o participar en una conversación práctica básica sobre un tema predecible.

$\checkmark$ Es capaz de comprender comunicaciones básicas, instrucciones o información.

$\checkmark \quad$ Puede completar formularios básicos y escribir notas que incluyen horas, fechas y lugares. (Press) [9]

De igual manera, el Ministerio de Educación de Ecuador destaca los estándares de calidad educativa del aprendizaje del Idioma Inglés dirigido a los estudiantes del nivel medio, lo cual está basado en cinco ámbitos del conocimiento, tales como:

$\checkmark$ Dominio especifico de la estructura del idioma.

$\checkmark$ Conocimiento de la cultura de los pueblos.

$\checkmark \quad$ Planificación de las normas basadas en inglés, uso de recursos didácticos y tecnológicos.

$\checkmark \quad$ La evaluación relacionada con el aprendizaje del idioma inglés.

$\checkmark$ Compromiso ético y académico que referencia el resultado de investigación en el campo de la enseñanza del idioma inglés. (Educación, 2014)

Sin embargo, a pesar de la existencia de estos estándares de calidad educativa del aprendizaje del idioma Inglés, continúa presentándose el desconocimiento del mismo por parte de los estudiantes que ingresan al Pre-Universitario, por ello se destaca la necesidad de continuar con la aplicación del Módulo Cero para quienes ingresan a este Centro de Educación Superior y se pueda medir el nivel de conocimiento previo a la ubicación respectiva de cada estudiante. (Maridueña, Jacqueline; Ledesma, Brigita \& Macías, Franklin, 2016) [10]

Como hipótesis general, este trabajo investigativo destaca:

$\checkmark \quad$ La incorporación del Módulo Cero como parte del aprendizaje del Idioma Inglés en los estudiantes que ingresan a la Universidad Estatal de Milagro repercute determinantemente en el aprendizaje y 
avance del conocimiento de esta lengua extranjera.

$\checkmark$ La incorporación del Módulo Cero influye significativamente en el interés de los estudiantes en relación al aprendizaje del idioma Inglés.

$\checkmark \quad$ Las actividades metodológicas que se desarrollan en Módulo Cero de Inglés permiten el desarrollo de las destrezas del idioma en los estudiantes que cursan los módulos de inglés.

$\checkmark$ La práctica de las destrezas del idioma inglés, impacta positivamente en el interés que los educandos demuestran en el desarrollo de las mismas.

De igual manera, se establecen los objetivos específicos de este documento:

$\checkmark$ Verificar las causas que generan el desinterés, reprobación y abandono de la asignatura de Inglés en los estudiantes que cursan los diferentes módulos de Inglés.

$\checkmark$ Incorporar actividades metodológicas que impulsen el desarrollo de las destrezas del idioma en el Módulo Cero de Inglés en los estudiantes.

$\checkmark$ Analizar los beneficios que los estudiantes que cursan el Módulo 1 de inglés han obtenido luego de haber realizado el Módulo Cero.

De acuerdo a Richard, Jack \& Renandya Willy (2008), se considera necesaria la recepción del test diagnóstico para conocer el nivel del idioma antes de dar paso a la planificación que conlleve a la preparación académica de los alumnos, lo cual permite obtener resultados satisfactorios ya que estos desarrollan las destrezas del idioma de manera correcta. (Richard, Jack \& Renandya, Willy, 2008) [11]

Según Jordan, R.R., los niveles de aprendizaje del idioma inglés dirigen la planificación del programa de estudio acorde con los requerimientos de los estudiantes. Esto hace que existe un aprendizaje significativo y estandarizado. (Jordan, 2012) [12]

Para Gill, Sara \& Adult Basic Education Association (2014), destaca la importancia de la evaluación formativa ya que mide los conocimientos y habilidades actuales de los alumnos con el fin de identificar a un plan de entrenamiento. (Ministry of Education, 2008) [13]

En este tipo de examen no se ha considerado la destreza de la expresión oral (speaking) debido a que resulta elocuente verificar con este tipo de examen escrito, si los alumnos tienen o no conocimientos del idioma inglés que sirva de soporte para ubicarlos en el nivel respectivo. (Real, Iliana \& Maridueña Jacqueline, 2016) [14]

Asimismo, las autoras consideraron el $20 \%$ de los estudiantes que realizaron el Módulo Cero en el Primer Semestre 2015 como la muestra, receptándoseles una encuesta enfocada de 10 preguntas referente a lo relacionado al Módulo Cero o Nivel de inicio. Es importante señalar que 65 estudiantes corresponden al $20 \%$, quienes estuvieron inmersos en este proceso de estudio, es decir, avanzaron con el módulo cero hasta el final. Esto significa, de 325 (universo), se consideró a 65 de ellos en calidad de muestra. (Milagro, sga.unemi, 2015)

\section{Metodología}

A continuación se detalla la información referente a los resultados de las encuestas realizadas a los estudiantes que aprobaron el Módulo Cero de Inglés en el Primer Semestre 2015. (Noguera, 2012) [15]

Para este proceso de investigación se utilizaron los métodos cuantitativo y cualitativo empleando un formato de encuesta que contempló 10 preguntas la misma que fue considerada parte fundamental para la elaboración de la presente investigación. Se realizó la encuesta a 65 estudiantes del Primer Semestre 2015 como muestra para evidenciar el resultado de este trabajo.

\section{Resultados}

Se presentan los resultados obtenidos mediante la aplicación de encuestas dirigidas a los estudiantes que tomaron el Módulo Cero de Inglés. Esta investigación da énfasis en los métodos cuantitativo y cuantitativo lo cual se ve reflejado en tablas y gráficos estadísticos. De igual manera se consideró la estadística descriptiva para determinar el valor considerado como muestra en el presente artículo. Este resultado demuestra claramente el impacto positivo que brinda el Módulo Cero en torno al aprendizaje del Idioma Inglés como lengua extranjera.

Seguidamente se detalla la información de mayor relevancia:

Pregunta 1. ¿Le agradó el Módulo Cero de Inglés?

Tabla 5. Agrado del Módulo Cero de Inglés

\begin{tabular}{|l|c|}
\hline ALTERNATIVA & FRECUENCIA \\
\hline Sí & 65 \\
\hline No & 0 \\
\hline TOTAL & 65 \\
\hline
\end{tabular}

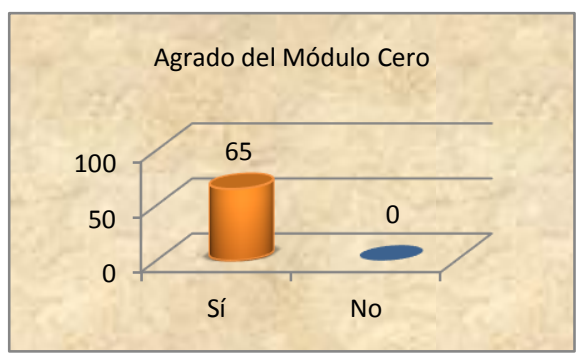

Figura 6. Agrado del Módulo Cero Fuente: Encuesta realizada a los estudiantes del Módulo Inglés I 
En la pregunta 1, los 65 encuestados indicaron que les agradó el Módulo Cero de Inglés, es decir, que existió total acogida por parte de ellos y eso hace pensar que desarrollaron las destrezas de esta lengua extranjera, demostrando interés en la asignatura y a su vez, reflejando la necesidad de este programa de estudio.

Pregunta 3. ¿Qué nivel de importancia representa para usted el aprendizaje del idioma Inglés?

Tabla 6. Importancia del aprendizaje del idioma Inglés

\begin{tabular}{|l|c|}
\hline ALTERNATIVA & FRECUENCIA \\
\hline Muy alto & 33 \\
\hline Alto & 23 \\
\hline Medio & 9 \\
\hline Bajo & 0 \\
\hline TOTAL & 65 \\
\hline
\end{tabular}

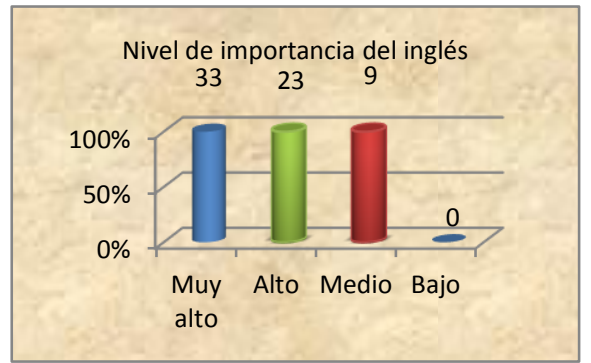

Figura 7. Nivel de importancia del Inglés

Fuente: Encuesta realizada a los estudiantes del Módulo

Inglés I

En la pregunta 3, 56 de los estudiantes manifestaron la importancia que para ellos representó la asignatura de Inglés, considerando la necesidad de adquirir este idioma como lengua extranjera a fin de que sirva como parte de su proceso educativo hasta cumplir los módulos reglamentarios de Inglés.

Pregunta 5. ¿Cree usted que con el Módulo Cero, el Módulo 1 de Inglés le será más práctico?

Tabla 7. Practicidad del Módulo Uno

\begin{tabular}{|c|c|}
\hline ALTERNATIVA & FRECUENCIA \\
\hline Sí & 64 \\
\hline No & 1 \\
\hline TOTAL & 65 \\
\hline
\end{tabular}

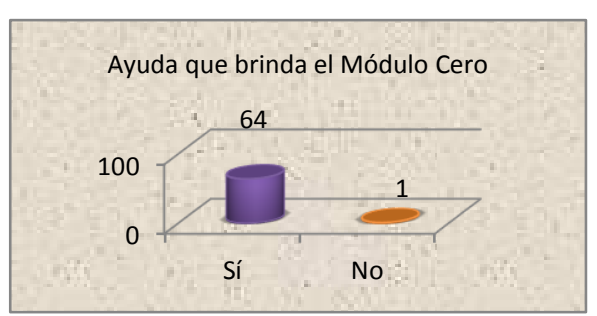

Figura 8. Ayuda del Módulo Cero

Fuente: Encuesta realizada a los estudiantes del Módulo Inglés I
En esta pregunta, apenas 1 estudiante indicó que el aprendizaje del Inglés no le era práctico si no realizara el Módulo Cero, esto brinda la evidencia de que los estudiantes en su mayoría aceptan la importancia de contar con este módulo inicial previo al Módulo 1, puesto que les ayuda a mejorar y comprender el idioma Inglés.

Pregunta 7. ¿Considera usted que la UNEMI debería continuar facilitando el Módulo Cero a los estudiantes que ingresan al Primer Semestre?

Tabla 8. Continuidad de facilitar el Módulo Cero

\begin{tabular}{|l|c|}
\hline ALTERNATIVA & FRECUENCIA \\
\hline Sí & 65 \\
\hline No & 0 \\
\hline TOTAL & 65 \\
\hline \multicolumn{2}{|c|}{ Se debe continuar con el Módulo Cero } \\
\\
\end{tabular}

Figura 9. Continuar con el Módulo Cero

Fuente: Encuesta realizada a los estudiantes del Módulo Inglés I En la pregunta 7, los encuestados en su totalidad indicaron que la Universidad Estatal de Milagro debería continuar brindando el Módulo Cero como parte del proceso de aprendizaje del Idioma Inglés a beneficio de los estudiantes.

Pregunta 9. ¿Con que frecuencia usted practica las diferentes destrezas del idioma Inglés (Listening, Speaking, Reading and Writing) como parte de su aprendizaje autónomo?

Tabla 9. Frecuencia en la práctica de las destrezas del idioma Inglés

\begin{tabular}{|l|c|}
\hline ALTERNATIVA & FRECUENCIA \\
\hline Siempre & 3 \\
\hline Frecuentemente & 12 \\
\hline Algunas veces & 35 \\
\hline Ocasionalmente & 14 \\
\hline Nunca & 1 \\
\hline TOTAL & 65 \\
\hline
\end{tabular}

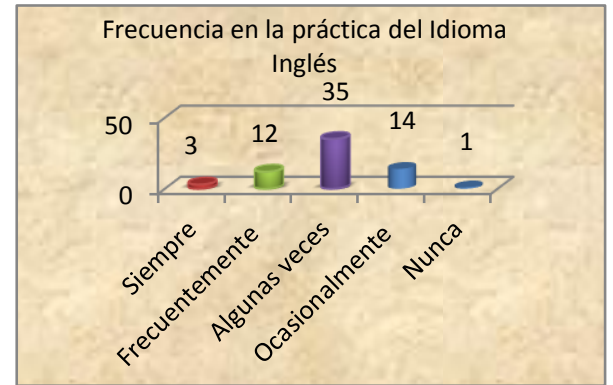

Figura 10. Frecuencia en la práctica del Idioma Inglés 
En la pregunta 9, 15 de los estudiantes indicaron que practican con frecuencia las destrezas del idioma Inglés y 35 de ellos sostuvieron que realizan esta tarea algunas veces. Esto demuestra que si existió el interés en los educandos que tomaron el Módulo Cero. En esta pregunta, se verificó también que apenas 15 encuestados no desarrollaron la práctica del idioma con frecuencia.

Pregunta 10. ¿Qué grado de importancia considera usted que tiene el aprobar los módulos reglamentarios de Inglés que se dictan en la Universidad Estatal de Milagro?

Tabla 10. Importancia en aprobar los módulos de Inglés

\begin{tabular}{|l|c|}
\hline ALTERNATIVA & FRECUENCIA \\
\hline Muy alto & 33 \\
\hline Alto & 29 \\
\hline Medio & 2 \\
\hline Bajo & 1 \\
\hline TOTAL & 65 \\
\hline
\end{tabular}

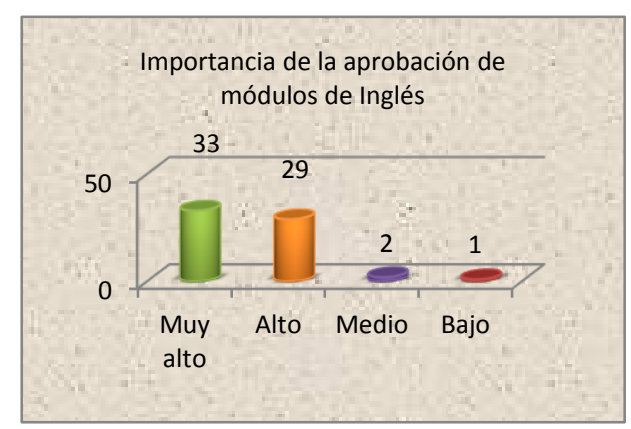

Figura 11. Importancia de la aprobación de módulos de Inglés

Fuente: Encuesta realizada a los estudiantes del Módulo Inglés I

En la pregunta 10, 62 encuestados manifestaron que están conscientes de la importancia que representa el aprobar los módulos e Inglés reglamentarios que se dictan en la Universidad Estatal de Milagro, como parte de su programa de estudio académico y porque constituye requisito previo para su egreso.

\section{Discusiones}

De acuerdo a las estimaciones presentadas por el British Council en relación al idioma inglés se estima que en el año 2016 aproximadamente 2000 millones de personas en el mundo entero estarán haciendo uso de este idioma como lengua extranjera, lo cual conlleva a comprender el grado de importancia que para los estudiantes debe tener la aprobación de los módulos reglamentarios de inglés y el desenvolvimiento oral y escrito del mismo. (School, 2010). [16] Vale la pena resaltar, que los estudiantes que participaron y aprobaron el Módulo Cero, notaron la importancia de contar con esta puerta de inicio del aprendizaje del idioma inglés. (Maridueña Macancela, Jacqueline \& Ledesma Acosta, Brigita, 2015) [17]

En la pregunta 3, el 99\% de los encuestados destacaron que el Módulo Cero representa un soporte de gran importancia para el avance de los siguientes módulos, lo cual ayuda a identificar que la Universidad Estatal de Milagro está dirigiendo la preparación y aprendizaje del inglés de manera correcta a beneficio de los estudiantes. Paralelamente, los resultados de la pregunta 7 corroboran la existencia de una total relación entre las mismas.

Estos datos detallan la dispersión referencial que se ve reflejada en el desarrollo del Módulo Cero dentro del proceso de aprendizaje del idioma Inglés, de quienes ingresan a la Universidad Estatal de Milagro.

\section{Conclusiones}

El presente trabajo investigativo permite conocer el grado de interés y de motivación que demuestran los estudiantes con la inclusión del Módulo Cero dentro del programa de estudio del Idioma Inglés, lo cual brinda satisfacción a los docentes de esta asignatura al momento de verificar los resultados que se encuentran evidenciados en las actas de calificaciones de los estudiantes y por ende, se reduce el porcentaje de alumnos que abandonan o reprueban los módulos de Inglés. Esto hace sentir mucha expectativa en un futuro cercano, debido a que la Universidad Estatal de Milagro debe dar cumplimiento a lo que dispone la Ley Orgánica de Educación Superior en su Art. 124 en concordancia con el Art. 30 de lo que indica el Consejo de Educación Superior; esto es que los estudiantes deban rendir una prueba de suficiencia en el Idioma Inglés luego de haber cursado y aprobado el $60 \%$ de las asignaturas de la carrera y así puedan obtener una Certificación Internacional en este idioma extranjero. (Superior, Consejo de Educación Superior, 2014) El alumno que necesita empezar sus estudios de Inglés con el Módulo Cero, lo está haciendo con paso firme. [18]

\section{Agradecimientos}

Se destaca la ayuda obtenida por parte de la Universidad Estatal de Milagro referente a las Resoluciones del Órgano Colegiado Académico Superior, lo cual ha permitido evidenciar el presente trabajo.

De igual manera, se debe considerar la información importante que se desprende del sistema de gestión académicasga@unemi.edu.ec , que permitió obtener los datos precisos de los docentes, estudiantes, módulos abiertos y actas de calificaciones como parte destacada de este proyecto. Así también, vale la pena mencionar el aporte brindado por las autoras de este documento, basado en las experiencias adquiridas y plasmadas en otros temas investigativos relacionados 
al mismo enfoque educativo y que hacen referencia a la importancia del aprendizaje del idioma inglés.

Finalmente, es importante resaltar que la preparación del manuscrito ha sido un proceso pormenorizado y bien específico que contempla el seguimiento de cuatro periodos académicos para referenciar el éxito de contar con la impartición del módulo Cero o de Inicio como soporte a aquellos estudiantes que ingresan a

\section{Referencias}

[1] Superior, C. d. (2013). Recuperado el 20 de 05 de 2015, de Consejo de Educación Superior República del Ecuador: http://www.ces.gob.ec/2012-10-29-08-5125/2012-10-29-08-51-54?start=20

[2] Uribe Zirene, J. d. (02 de 2012). Importancia del idioma inglés en las instituciones de educación superior: el caso de la Corporación Universitaria de Sabaneta. Uni-Pluriversidad, 12(2).

[3] Maridueña Macancela, J. (2014). Recursos Interactivo Tecnológicos y su repercusión en el Interaprendizaje del Idioma Inglés . RES NON VERBA, 1-18.

[4] Milagro, U. E. (2014). Recuperado el 27 de 11 de 2014, de Resoluciones OCAS 2014 UNEMI:

http://www.unemi.edu.ec/web/images/pdf/R esolucionesOCAS/14ocas-so-14-2014.pdf

[5] Latham-Koening C, Oxenden C, y Seligson P,. (2013). American English File Starter Teacher's book, second edition. London: Oxford University Press.

[6] Milagro, U. E. (2015). Recuperado el 15 de 10 de 2015, de sga.unemi: http://sga.unemi.edu.ec/adm_docentes

[7] Fleta Guillén, T. (2006). Aprendizaje y técnicas de enseñanza del inglés en la escuela. 16(2).

[8] Navas Yonaiker; Real Iliana; Pacheco Silvia. (2016). Los procesos de enseñanza y aprendizaje del idioma inglés a través de los entornos virtuales de aprendizaje. $8(13)$.

[9] Assessment, C. E. (2015). Recuperado el 15 de 10 de 2015, de Cambridge English: http://www.cambridgeenglish.org/whycambridge-english/international-languagestandards/

[10] Press, C. U. (s.f.). Recuperado el 20 de 01 de 2015, de Cambridge English Language Assessment: esta universidad y que vienen de establecimientos educativos de nivel medio con alto nivel de desconocimiento del idioma Inglés, lo cual constituye una fortaleza poder brindarles este servicio que les ayuda en el aprendizaje del inglés como lengua extranjera.

http://www.cambridgeenglish.org/es/exams/ cefr/

[11] Educación, M. d. (2014). Recuperado el 06 de 10 de 2015, de Estándares de calidad educativa: http://educacion.gob.ec/wpcontent/uploads/downloads/2012/09/estanda res_2012_ingles_opt.pdf

[12] Maridueña, Jacqueline; Ledesma, Brigita \& Macías, Franklin. (2016). Módulos de Inglés y su repercusión en las destrezas del idioma. 9(19).

[13] Richard, Jack \& Renandya, Willy. (2008). Methodology in Language Teaching (11 ed.). (C. U. Press, Ed.) New York: Cambridge University Press.

[14] Jordan, R. (2012). English for Academic Purposes (13 ed.). (C. U. Press, Ed.) Cambridge: Cambridge University Press.

[15] Ministry of Education, M. o. (Ed.). (2008). Canadian and World Research and Assessment Practices for Adult Learners. Canada: Project READ Literacy Network (PRLN).

[16] Real, Iliana \& Maridueña Jacqueline. (2016). Reflexión de la relación que existe entre la pronunciación, la comprensión auditiva y la expresión oral en la lengua inglesa. 4(1).

[17] Noguera, M. (2012). Recursos digitalizados de Apoyo didáctico para la enseñanza del esquema discursivo del inglés en la Modalidad Semipresencial, a Nivel universitario. 6(2).

[18] School, E. B. (2010). Recuperado el 02 de 10 de 2015, de Euroinnova Business School : http://www.euroinnova.edu.es/

[19] Maridueña Macancela, Jacqueline \& Ledesma Acosta, Brigita. (2015). Orientación del Trabajo Independiente y el Uso de las TIC. Revista Científica Ciencia - UNEMI, 1-9.

[20] Superior, C. d. (2014). Consejo de Educación Superior. Recuperado el 20 de 08 de 2015, de http://www.ces.gob.ec 